\title{
Actuation Principles for Hybrid Two-Dimensional Quasistatic Micro Scanning Mirrors
}

\author{
S. Kimme ${ }^{1}$, Th. Sandner ${ }^{1}$, A. Graf ${ }^{1}$, U. Todt ${ }^{1}$, J. Czarske ${ }^{2}$ \\ ${ }^{1}$ Fraunhofer IPMS, Maria-Reiche-Str. 2, 01109 Dresden, Germany \\ simon.kimme@ipms.fraunhofer.de \\ 2 Professur für Mess- und Prüftechnik, TU Dresden, Helmholtzstr. 18, 01069 Dresden, Germany
}

\begin{abstract}
In this article a numerical approach for a hybrid actuated two-dimensional micro scanner with large aperture is presented for applications like 3D distance measurement and high power laser applications like laser material treatment or medical laser surgery. No micromachined mirror with large aperture $(>\varnothing 6 \mathrm{~mm})$ and large quasistatic deflection $\left(>2^{\circ}\right)$ is known. Only fine mechanically fabricated scanners reach large deflection angels with, in comparison to micromirrors, huge aperture [1,2]. Our approach is, to combine a micromachined structure with an external actuator. To be able to choose the best suitable actuator, an analytical design model was developed. Optimizations of a magnetic actuator guiding the magnetic flux are presented and evaluated experimentally with a gimbal micromirror with $6 \mathrm{~mm} \times 8 \mathrm{~mm}$ mirror plate. Static deflections of $3^{\circ}$ were reached.
\end{abstract}

Key words: quasistatic scanning micromirror, large aperture, two-dimensional, hybrid actuation, high power application

\section{Introduction}

Miniaturized active optical elements like micromechanical scanning mirrors are successfully applied in many optical application fields like Fourier transform infrared spectroscopy, time of flight distance measurements and image projection. Vertical comb drives [3] are easy to integrate in micromechanical fabrication processes and allow a quasi-static positioning but they are limited due to the scaling-laws of electrostatic actuation and can achieve only small actuation forces resulting in small deflection angles for larger mirror diameters of several milimeters. Further "macro-"mechanical approaches like in [1] try to miniaturize a system with a classically mounted and fabricated mirror platform. However relatively huge afford has to be undertaken to fabricate this device.

\section{State of the art}

Some scanning devices with different actuation principles are posed in tab. 1. The maximum mechanical scan angle $\hat{\Theta}_{\text {mech }}$, the area of the mirror plane $A$ and the resonant frequencies $f$ are compared. Only fine mechanically fabricated scanners reach large deflection angles with, in comparison to micromirrors, huge aperture [2].
Tab. 1: Review of quasistatic actuated micro mirrors. Quasistatic characteristic values are shown bold. "Ø" stands for the diameter of a circular mirror plane, " $x$ " in between side lengths of rectangular planes.

\begin{tabular}{|c|c|c|c|c|}
\hline $\begin{array}{l}\stackrel{\square}{\Phi} \\
\simeq\end{array}$ & 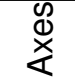 & $\hat{\Theta}_{\text {mech }} /^{\circ}$ & $A / \mathrm{mm}^{2}$ & $f / \mathrm{kHz}$ \\
\hline \multicolumn{5}{|c|}{ Electrostatic actuation } \\
\hline [4] & 2 & $6 / 7$ & $\varnothing 1$ & $0,14 / 0,46$ \\
\hline [5] & 2 & 9/10 & $\varnothing 0,6$ & $4,6 / 4,6$ \\
\hline \multicolumn{5}{|c|}{ Piezoelectric actuation } \\
\hline [6] & 2 & $0,3 / 0,25$ & $5 \times 5$ & $0,03 / 0,09$ \\
\hline [7] & 2 & $8,6 / 5,5$ & $1 \times 1,5$ & $0,43 / 1,52$ \\
\hline \multicolumn{5}{|c|}{ Thermal actuation } \\
\hline [8] & 2 & $2,7 / 2,6$ & $\varnothing 1$ & ca. 0,01 \\
\hline [9] & 2 & $4 / 4$ & $\varnothing 0,6$ & $0,04 / 0,04$ \\
\hline \multicolumn{5}{|c|}{ Electromagnetic actuation } \\
\hline [10] & $1 \& 1$ & $22 / 0,5$ & $\varnothing 1,5$ & $0,4 / 22,2$ \\
\hline [2] & 2 & $2,5 / 3$ & $\varnothing 40$ & 0,12 \\
\hline \multicolumn{5}{|c|}{ Hybrid actuation } \\
\hline [11] & $1 \& 1$ & $10 / 12$ & $\varnothing 1,2$ & $1,58 / 3,05$ \\
\hline
\end{tabular}

\section{Choice of actuator, design model}

There are many aspects that have to be taken into account to rate different actuation principles. The most significant are the moving range, the attainable force and the dynamic of an actuator. 
Tab. 2: Several actuation principles using electromagnetic forces and the related laws of force calculation used in the design model.

\begin{tabular}{|c|c|c|c|}
\hline Schematic illustration & Physical relationships & Working range & Scoring \\
\hline \multicolumn{4}{|l|}{ Electrodynamic actuator } \\
\hline \multirow{2}{*}{$\mathrm{N} \underbrace{N, I_{\mathrm{W}}}_{\leftarrow 2 r_{\mathrm{c} \rightarrow}}{ }^{B}{ }^{B} \mathrm{~S}$} & \multirow[t]{2}{*}{$\begin{array}{l}F\left(I_{\mathrm{A}}\right)=B 2 \pi r_{\mathrm{c}} N I_{\mathrm{A}} \\
\text { with } \\
B \ldots \text { magn. flux density } \\
r_{\mathrm{c}} \ldots \text { radios of the coil } \\
N \ldots \text { number of turns }\end{array}$} & \multirow[t]{2}{*}{ 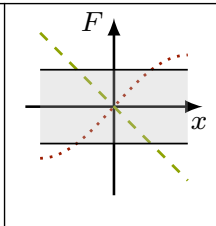 } & $\begin{array}{l}+ \text { force not dependent of deflec- } \\
\text { tion } \\
+ \text { bidirectional force action }\end{array}$ \\
\hline & & & $\begin{array}{l}\text { - electrically active component } \\
\text { moved }\end{array}$ \\
\hline \multicolumn{4}{|c|}{ Electromagnetic actuator with flat coil } \\
\hline \multirow{2}{*}{ 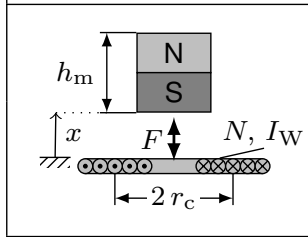 } & \multirow{2}{*}{$\begin{array}{l}F\left(x, I_{\mathrm{A}}\right)=B_{\mathrm{r}} A_{\mathrm{m}} \int_{x}^{x+h_{\mathrm{m}}} \frac{\partial H_{x}\left(x, I_{\mathrm{A}}\right)}{\partial x} d x \\
H\left(x, I_{\mathrm{A}}\right)=\frac{N I_{\mathrm{A}} r_{c}^{2}}{2\left(r_{\mathrm{c}}^{2}+x^{2}\right)^{\frac{3}{2}}} \\
\begin{array}{c}\text { with } \\
H \ldots \text { field intensity } \\
B_{\mathrm{r}} \ldots \text { remanent flux density } h_{\mathrm{m}} \ldots \text { height of magnet } \\
r_{\mathrm{m}} \ldots \text {. footprint of magnet }\end{array}\end{array}$} & & $\begin{array}{l}+ \text { high energy densities } \\
+ \text { flat coils easy integrable }\end{array}$ \\
\hline & & & $\begin{array}{l}\text { - nonlinear force characteristic } \\
\text { - mutual actuation necessary } \\
\text { - thus asymmetric load }\end{array}$ \\
\hline \multicolumn{4}{|c|}{ Electromagnetic actuation with cylindrical coil } \\
\hline \multirow{2}{*}{ 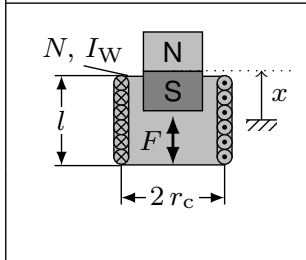 } & \multirow{2}{*}{$\begin{array}{l}F\left(x, I_{\mathrm{A}}\right)=B_{\mathrm{r}} A_{\mathrm{m}} \int_{x-\frac{h_{\mathrm{m}}}{2}}^{x+\frac{h_{\mathrm{m}}}{2}} \frac{\partial H_{x}\left(x, I_{\mathrm{A}}\right)}{\partial x} d x \\
H\left(x, I_{\mathrm{A}}\right)=\frac{N I_{\mathrm{A}}}{2 l}\left(\cos \alpha_{1}-\cos \alpha_{2}\right) \\
\text { with } \\
\alpha_{1}=\arctan \frac{r_{\mathrm{C}}}{\frac{l}{2}+x}, \quad \alpha_{2}=\pi-\arctan \frac{r_{\mathrm{C}}}{\frac{l}{2}-x} \\
\text { further symbols see actuator with flat coil }\end{array}$} & & $\begin{array}{l}+ \text { nearly constant actuation force } \\
+ \text { high energy density } \\
+ \text { symmetric bidirectional actua- } \\
\text { tion achievable }\end{array}$ \\
\hline & & & $\begin{array}{l}\text { - minimum force at maximum of } \\
\text { deflection }\end{array}$ \\
\hline \multicolumn{4}{|c|}{ 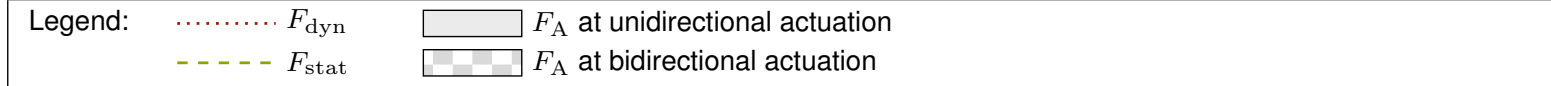 } \\
\hline
\end{tabular}

For instance piezoelectric actuators are limited by their very small moving range, piezoelectric stepper motors have an unlimited moving range and provide a sufficient high force, but they are limited in speed and resolution. Another aspect is the possibly needed additional mass at the mirror. Actuators with a moving magnet are therefore unsuitable for higher frequencies.

To consider the limits due to the actuation a simplified model of an one-dimensional rotating mirror is used. A sketch is shown in fig. 1. It is assumed, that the acquired insights can be interpreted for the two-dimensional case as well. For a given actuation frequency the equilibrium of torques is calculated:

$$
0=k\left(J, m_{\mathrm{A}}, l, f\right) \cdot \Theta-F_{\mathrm{A}}(\Theta, l) \cdot l
$$

In this nonlinear equation the relation behind the term $F_{\mathrm{A}}(\Theta, l)$ depends on the chosen actuation principle that is characterized by analytical relationships. The specific relationships depend on the constructive realization. Some opportunities for electromagnetic actuation are depicted in tab. 2.

The electrodynamic called actuation, describing the force on a current-carrying coil in a constant magnetic field is not advantageous for micromir-

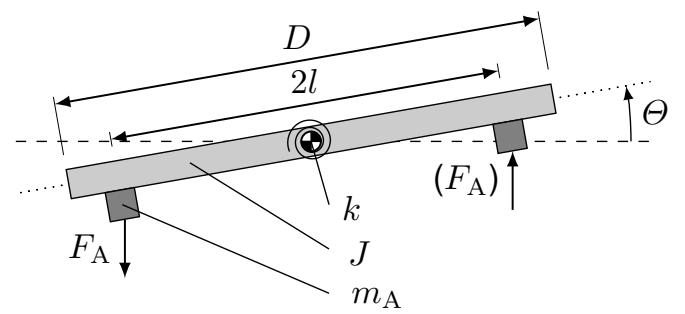

Fig. 1: Model for estimation of actuation principles for scanning mirrors.

rors because of design reasons. A moving magnet with a flat coil can generate high forces, but only for a small moving range. Using a cylindrical coil with a dipping magnet relatively large forces can be generated over a wide moving range. However the strength of the magnetic field is smaller than with a flat coil, because a larger inner coil diameter is needed.

\section{Simulation results}

The results of the simulations regarding two magnetic and two piezoelectric actuators without any additional lever mechanisms are shown in fig. 2. Presented is in fig. 2(a) the maximum quasistatic scan angle for a mirror with the related resonant frequency (corresponding to the 

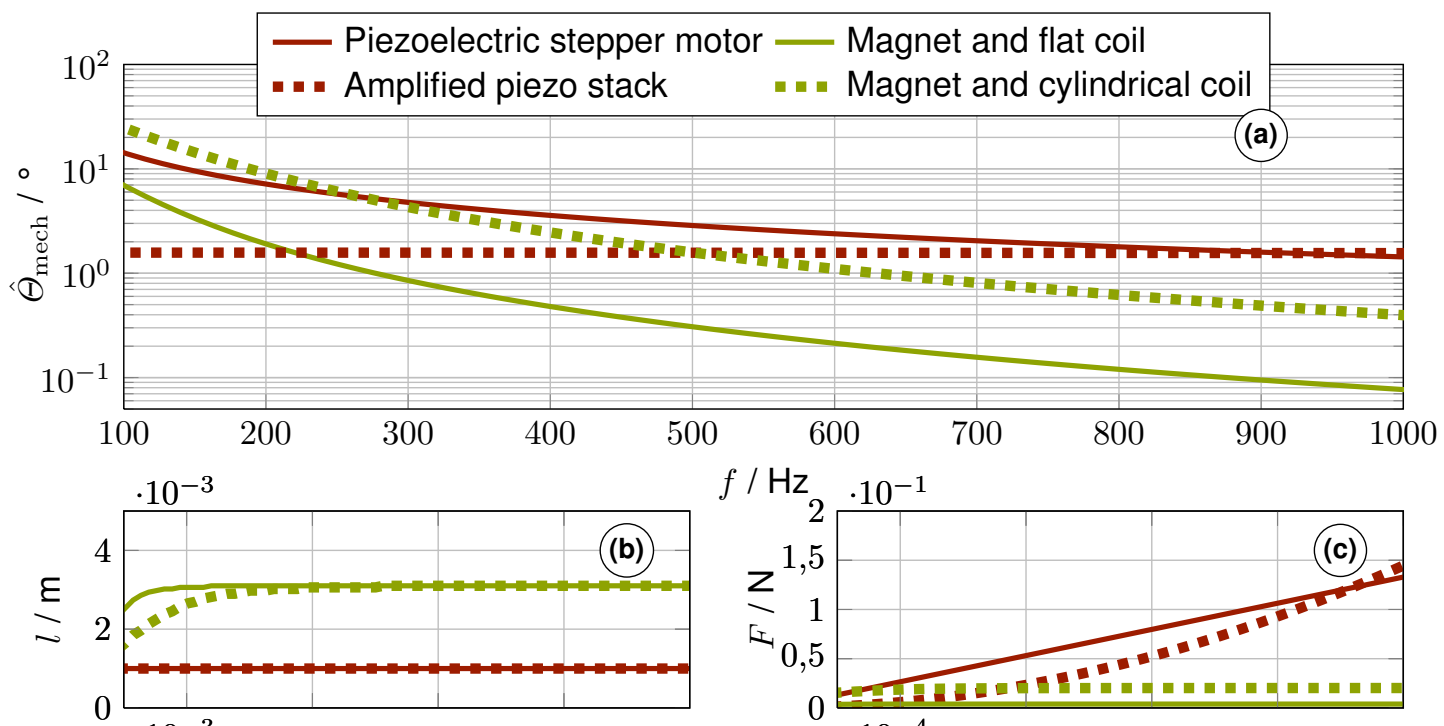

$$
f / \mathrm{Hz}_{2} \cdot 10^{-1}
$$
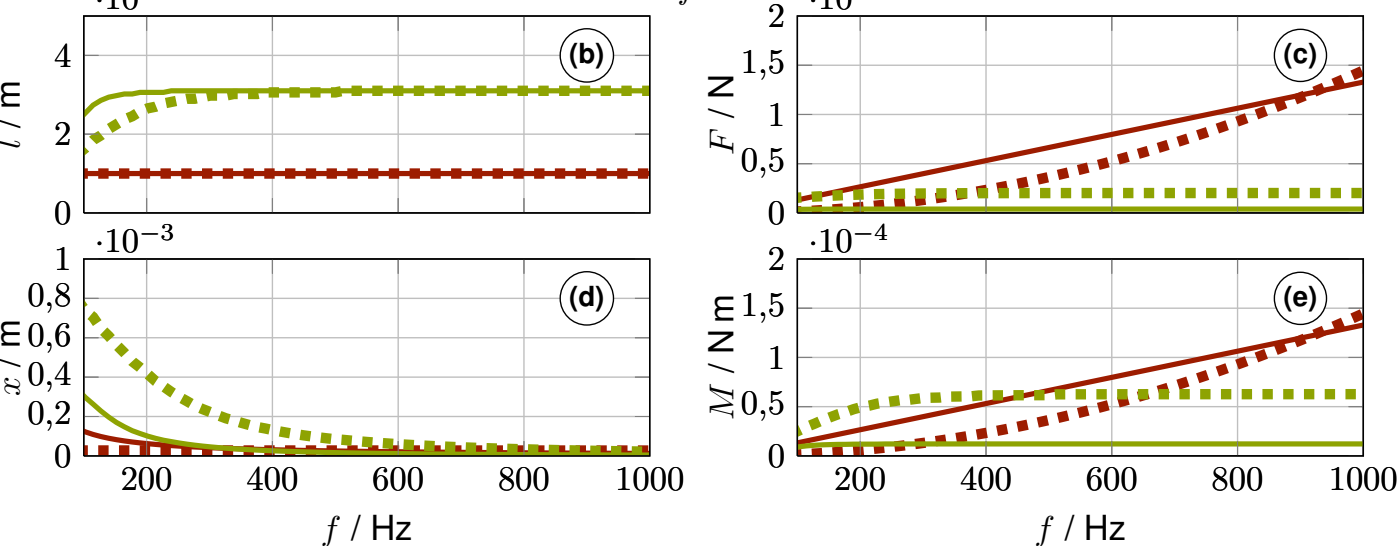

Fig. 2: Simulation results for maximum static deflection angle for different actuation principles: (a) maximum static deflection angle $\hat{\Theta}_{\text {mech }}$; (b) optimal distance $l ;$ (c) maximum force at maximum deflection $F ;(d)$ moving range $x ;(e)$ resulting torque $M$.

resulting inertia $J_{\text {res }}\left(J, l, m_{\mathrm{A}}\right)$ and stiffness $k$ of the flexure hinge). For low frequencies magnetic actuation with a cylindrical coil provides the best deflections. A piezoelectric stepper motor allows only low resolution due to relatively big steps of $1 \mu \mathrm{m}$ and a short lever (see fig. 2(b)). Actuation with a piezoelectric stack actuator shows, taking the resolution into account, the best results at frequencies higher than $500 \mathrm{~Hz}$. But without using lever mechanisms only very small deflection angles were achieved.

Due to the principal aim of large deflection angles requiring wide moving range electromagnetic actuation was choosen for further investigations.

\section{Optimized electromagnetic actuation}

The achieved forces of electromagnetic actuators with moving magnet depend, beside the permanent remanence of the magnet, on the gradient of the magnetic field intensity in the volume of the magnet. This can be increased by increasing the field intensity in general or by positioning the magnet exactly at the position of the maximum field gradient. The former can be reached by applying high current densities to the coil, reducing the inner diameter or (less effective) increasing height or outer diameter of the coil. This suggests the use of flat coils. The field gradient has it's maximum at the ends of the coil. Hence a movement of the magnet into the coil would be preferable. With the help of flux guiding material (FGM) both, small inner coil diameters and maximum field gradient at the position of the magnet over a wide moving range, can be achieved. In fig. 3 a cutaway view of a rotationally symmetric design with FGM is posed.

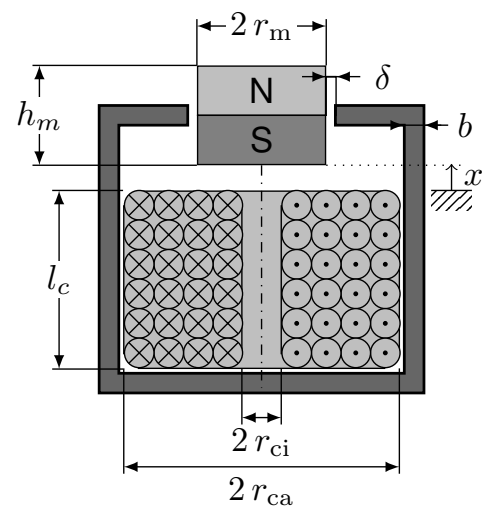

Fig. 3: Coil with magnetic flux guiding ferromagnetic material. 
As simulations with a finite element model showed, two additional force determining parameters accure. The gap $\delta$ between magnet and FGM influences the magnitude of the magnetic force. The thickness of the sheet $b$ has got even an influence on the direction of acting forces and the location of stable magnet positions. In fig. 4 the simulation results for force characteristics are shown for two different thicknesses $b$ with and without electrical current.

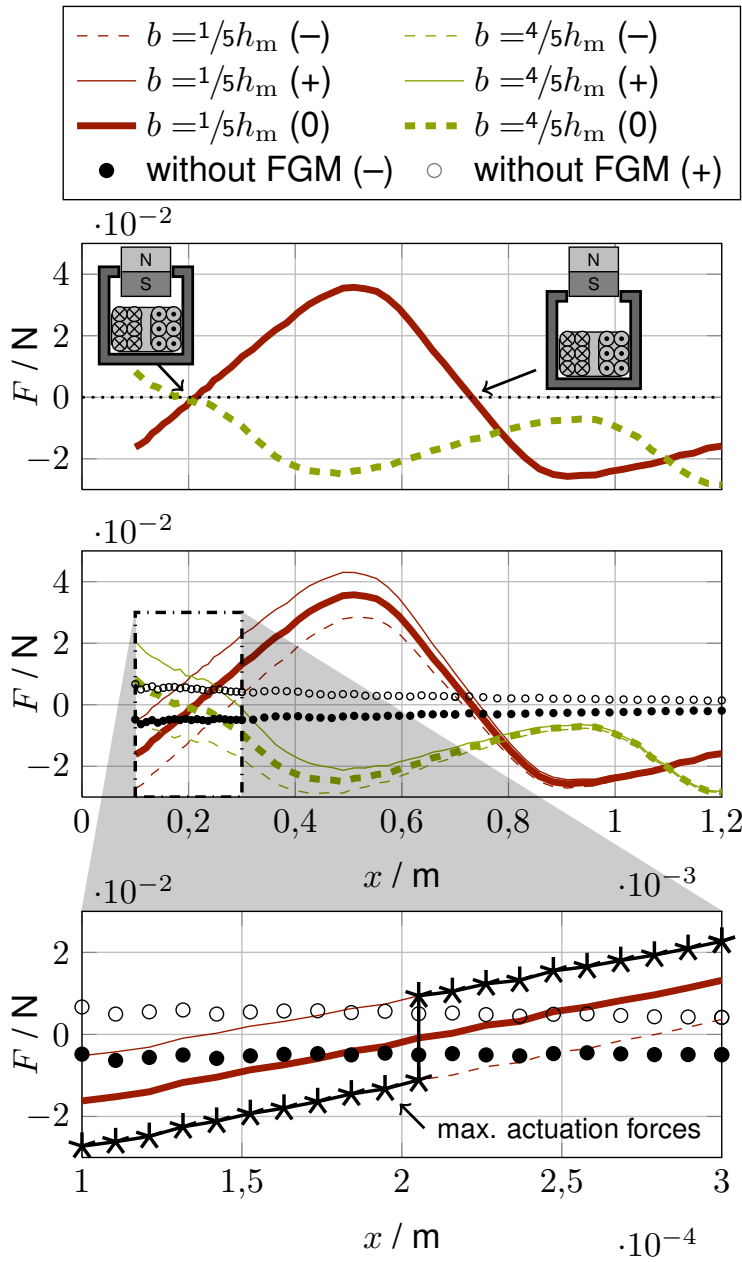

Fig. 4: Effects of thickness $b$ of the flux guiding material and the electrical current in the coil on the resulting electromagnetic forces. "- " and "+" label current with contrary orientation, " 0 " means "no current".

For slight sheets there exist two stable balance points (footprint of the magnet at middle of the sheet) and one unstable (center of the magnet at middle of the sheet). For thicker sheets only this latter balance piont is stable. This effect can be used to affect the actuation forces directed.

The detail view in fig. 5 shows raise of actuation force $\Delta F_{0}$ with FGM in the unstable balance point. The magnetic force pulling the magnet out of this unstable balance point can be described

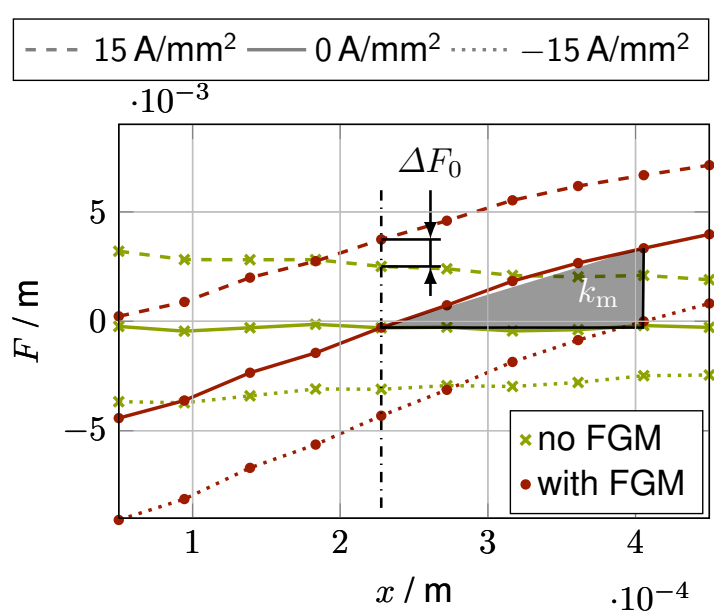

Fig. 5: Forces at the unstable balance piont with / without FGM for different current densities.

as a negative magnetic stiffness $k_{\mathrm{m}}$. For demonstration purposes a device was built and experimentally investigated.

\section{Mechanical characterization}

The tested device is shown in fig. 6 . On the backside of a gimbal mounted micromirror with a $75 \mu \mathrm{m}$ thick high reflective coated mirror plate of $6 \mathrm{~mm} \times 8 \mathrm{~mm}$ aperture four magnets with $1 \mathrm{~mm}$ diameter and $1 \mathrm{~mm}$ height made from $\mathrm{NdFeB}$ are glued. The static target-deflection is $2^{\circ}$. Examined are two actuator configurations without and with FGM $(b=200 \mu \mathrm{m}, \delta=500 \mu \mathrm{m})$. The coil dimensions are $r_{\mathrm{ca}}=2,25 \mathrm{~mm}, r_{\mathrm{ci}}=0,5 \mathrm{~mm}$ and $l_{\mathrm{c}}=5 \mathrm{~mm}$.

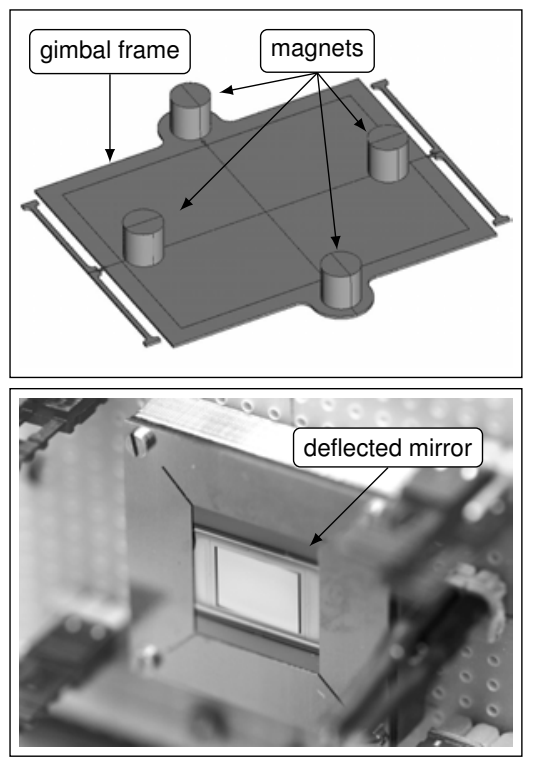

Fig. 6: Finite element model and photograph of the evaluated electromagnetic micro mirror

Characteristic of static deflection of the device is posed in fig. 7 . With the use of FGM about $50 \%$ 
higher deflection angles can be reached at identical current density. This reduces the heating of the coils which leads to undesired thermal drift due to decrease of the magnetization characterized by the reversible temperature coefficient of $\mathrm{NdFeB}$ (see fig. 8).

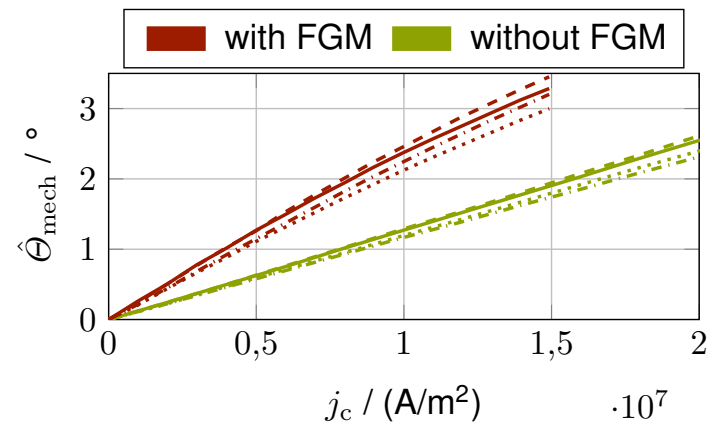

Fig. 7: Characteristic of static deflection of the mirror with and without FGM in all quadrants.

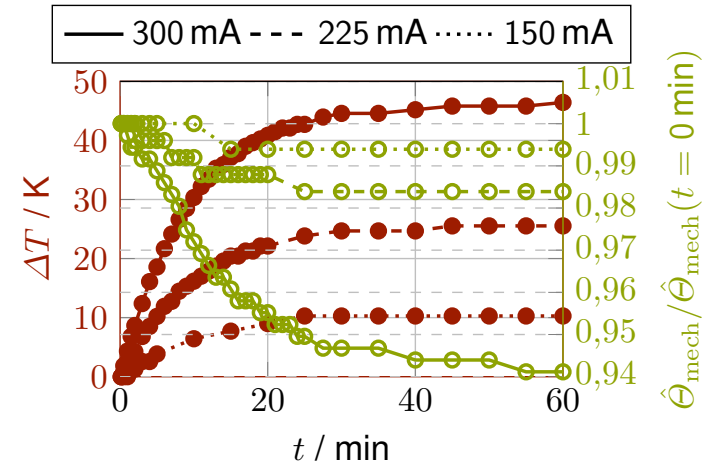

Fig. 8: Thermal effect on the deflection angle for different currents in the actuating coils.

The combined deflection of both axes shows no coupling. The measured deviations are less than the measurement accuracy (ca. 0,04\%).

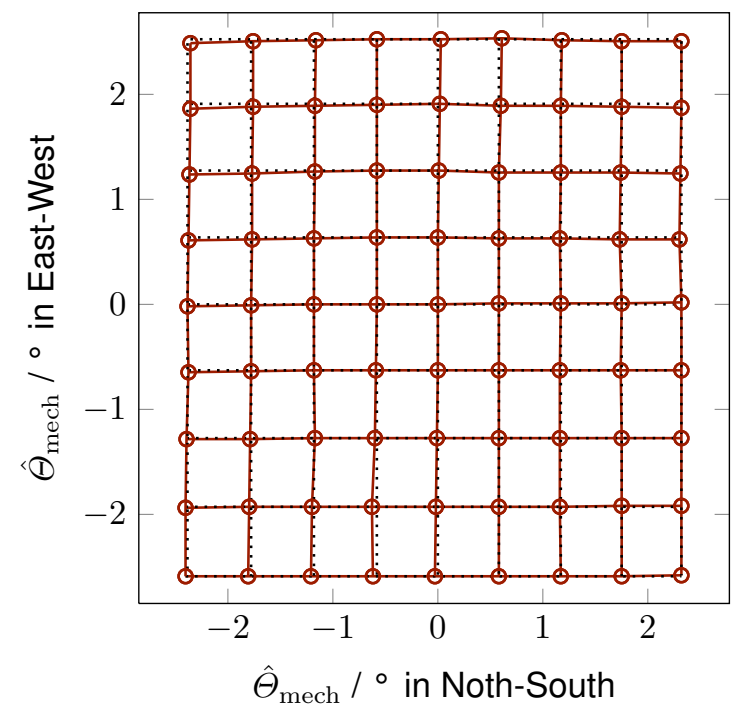

Fig. 9: Resulting deflection at simultaneous actuation of both axes.
The dynamic characterization with the help of analysis of free damped oscillation resulted in the resonant frequencies shown in fig. 10. The fall of the resonant frequencies with $F G M$ is attributed to the current-independent magnetic forces which take effect like a negative magnetic stiffness. Obvious from the graph of the decreasing vibration the damping coefficient with FGM is much bigger $\left(10 \mathrm{~s}^{-1}\right)$ than without $\left(6 \mathrm{~s}^{-1}\right)$. This is caused by eddy current occuring in the FGM due to the movement of the magnet.

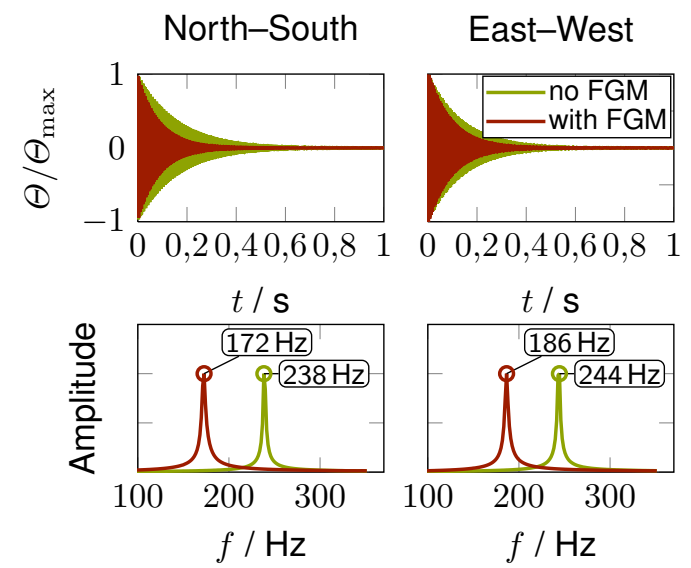

Fig. 10: Dynamic characterization by measurement of free damped oscillation for both axes with and without FGM.

\section{Optical characterization}

To enable high power laser applications in laser surgery the mirror plate is coated with a high reflective dielectric bragg coating. The coating design is temperature and stress compensated to guarantee a high optical flatness of the $75 \mu \mathrm{m}$ thick monocrystalline silicon mirror plate.

The flatness of the mirror plate was measured by use of white-light interferometry. The results for both, deflected and undeflected mirror, are shown in fig. 11. The curvature caused by the bending is only little effected by the deflection. The radius of curvature remains above $5 \mathrm{~m}$.

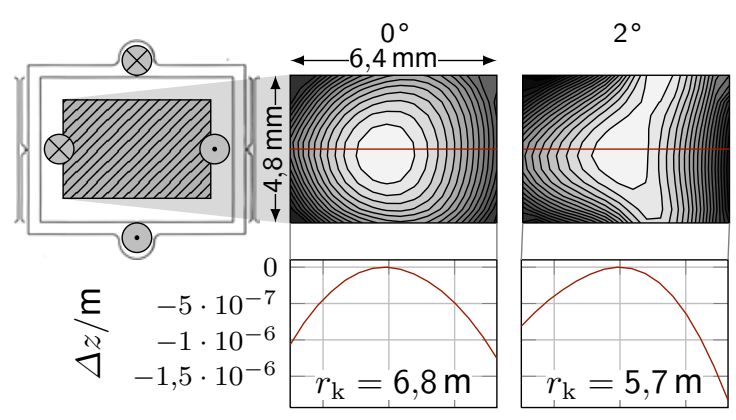

Fig. 11: Planarity of the mirror plate with and without deflection. The points of origin of force are posed on the right draft. 
Characteristics of the coating for an angle of incidence of $45^{\circ}$ are a reflectivity $\varrho \approx 99,9 \%$ for a wavelength of $532 \mathrm{~nm}$ (see fig. 12). The sustainable continuous power $P_{\mathrm{cw}}$ is $20 \mathrm{~W}$ at a pulse frequency of $20 \mathrm{kHz}$ and a pulse length of $15 \mathrm{ps}$.

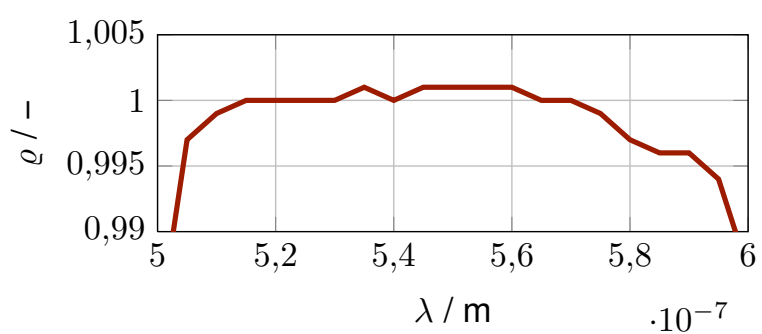

Fig. 12: Spectral reflectance $\varrho$ measured locally at the coated mirror plate at $45^{\circ}$ angle of entry using a spectral ellipsometer setup. The measurement uncertainty is estimated about $0,2 \%$.

For a rotating mirror a high reflectivity over a wide range of deflection is essential as well. That's why the reflectivity was measured for a deflection range of $30^{\circ}$. The results posed in fig. 13 show a reflectivity of over $99 \%$ at $\lambda=532 \mathrm{~nm}$ in this range.

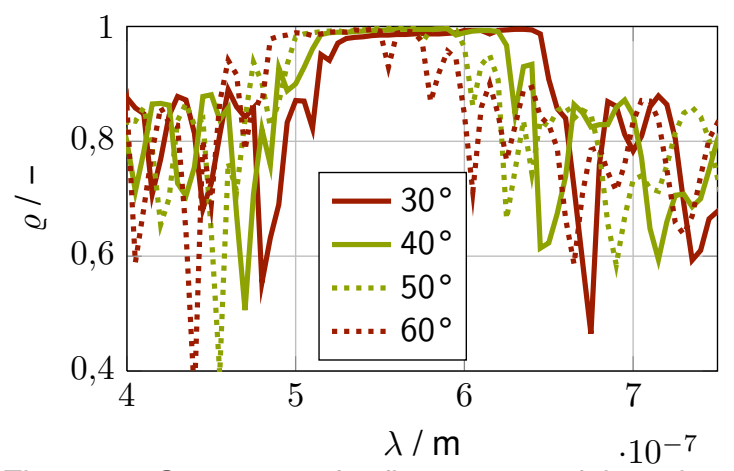

Fig. 13: Spectrum of reflectance $\varrho$ of the mirror coating over a range of angles of entry.

\section{Conclusion}

Actuation principles for micro mirrors with large aperture and hybrid actuation were investigated by simulations. The electromagnetic actuation has proved to be advantageous for large deflection angles without additional lever mechanisms. An actuator design with magnetic flux guiding material was designed and experimental examined. The predicted advantage of higher forces was verified as well as the disadvantage of lower resonant frequency. Nevertheless with the use of flux guiding material and corresponding design the dynamic characteristics were improved. Furthermore a faster positioning due to higher damping coefficients can be realized. The high reflective coating opens up possibilities for high energy applications.

\section{References}

[1] M. Hafez, Compact fast-steering tip/tilt laser scanner for high power material processing applications, PhD thesis, EPFL Lausanne, 2000, doi: 10.5075/epfl-thesis-2247

[2] S. Xiang et al., The Research of a novel single mirror 2D laser scanner, in: International Symposium on Photoelectric Detection and Imaging: Laser Sensing and Imaging, 2009

[3] D. Jung, Neuartiges Bauteilkonzept quasistatisch-resonanter 2D-Mikroscanner für die Zielanwendung ultrakompakter Laserprojektionsdisplays, PhD thesis, TU Dresden, 2012

[4] A. D. Aguirre et al., Two-axis MEMS Scanning Catheter for Ultrahigh Resolution Three-dimensional and En Face Imaging, in: Opt. Express 15.5 (Mar. 2007), pp. 2445-2453, doi: 10.1364/OE.15.002445

[5] V. Milanović; G. Matus, and D. McCormick, Gimbal-less monolithic silicon actuators for tip-tilt-piston micromirror applications, in: IEEE Journal of Selected Topics in Quantum Electronics 10.3 (June 2004), pp. 462-471, doi: 10.1109/JSTQE.2004.829205

[6] K. H. Koh et al., Novel piezoelectric actuation mechanism for a gimbal-less mirror in 2D raster scanning applications, in: Journal of Micro"-mechanics and Micro"-engineering 21.7 (2011)

[7] M. Tani et al., A two-axis piezoelectric tilting micromirror with a newly developed PZT-meandering actuator, in: IEEE 20th International Conference on Micro Electro Mechanical Systems, Jan. 2007, pp. 699-702, doi: 10.1109/MEMSYS.2007.4432994

[8] Y. Eun and J. Kim, Thermally driven torsional micromirrors using pre-bent torsion bar for large static angular displacement, in: Journal of Micromechanics and Microengineering 19.4 (2009)

[9] J. Singh et al., 3D free space thermally actuated micromirror device, in: Sensors and Actuators A: Physical 123-124.0 (2005), pp. 468-475, doi: 10.1016/j.sna.2005.02.037

[10] A. D. Yalcinkaya; H. Urey, and S. Holmstrom, NiFe Plated Biaxial MEMS Scanner for 2-D Imaging, in: IEEE Photonics Technology Letters 19.5 (Mar. 2007), pp. 330-332, doi: 10.1109/LPT.2007.891592

[11] L. Li et al., A symmetric hybrid MEMS scanner with electrothermal and electrostatic actuators, in: Optical MEMS and Nanophotonics (OMN), Aug. 2011, pp. 163-164, doi: 10.1109/OMEMS.2011.6031038 